\title{
Appareil
}

$20 \mid 2018$

Arts contemporains et patrimoine

\section{L'Hôtel-Dieu de Tonnerre : monument ou objet ?}

\section{Sylvie Le Clech-Charton}

\section{(2) OpenEdition}

Journals

Édition électronique

URL : http://journals.openedition.org/appareil/2737

DOI : 10.4000/appareil.2737

ISSN : 2101-0714

\section{Éditeur}

MSH Paris Nord

\section{Référence électronique}

Sylvie Le Clech-Charton, «L'Hôtel-Dieu de Tonnerre : monument ou objet ? », Appareil [En ligne], 20 | 2018, mis en ligne le 31 octobre 2018, consulté le 30 juillet 2020. URL : http://

journals.openedition.org/appareil/2737 ; DOI : https://doi.org/10.4000/appareil.2737

Ce document a été généré automatiquement le 30 juillet 2020.

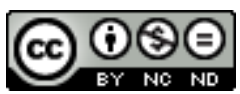

Appareil est mis à disposition selon les termes de la Licence Creative Commons Attribution - Pas d'Utilisation Commerciale - Pas de Modification 4.0 International. 


\title{
L'Hôtel-Dieu de Tonnerre : monument ou objet?
}

\author{
Sylvie Le Clech-Charton
}

\section{Fil conducteur}

1 Progressivement, l'Hôtel-Dieu, à la fois œuvre de charité douée d'une autonomie de gestion via une fondation due à la comtesse Marguerite de Tonnerre, et œuvre matérielle - un bâtiment dans son environnement domanial contenant des ensembles d'objets -, s'est affranchi des liens avec l'environnement immédiat pour devenir un objet à facettes qu'il est difficile de percevoir dans sa globalité.

2 Nous pourrions faire remonter cette dissociation de l'œuvre (ainsi définie) d'avec son environnement plus large et cette métamorphose au moment où le bâtiment et son domaine ont fait "patrimoine». Ce fait générateur s'associe alors à l'écriture de l'histoire et à des faits qui tiennent lieu de mémoire collective et individuelle. Il y a changement de point de vue et ce changement est servi par les avatars successifs du paysage monumental et foncier qui affecte l'Hôtel-Dieu.

3 En cela, la situation de l'Hôtel-Dieu de Tonnerre est particulière si on la compare à celle de Beaune qui a connu des adjonctions et des évolutions dans l'usage, mais jamais de destructions, ou à d'autres hôpitaux qui ont très longtemps gardé leurs fonctions (Louhans jusque dans les années 1990, encore maintenant Châlons ou Mâcon, véritables complexes hospitaliers). Dans ces hôpitaux, le discours monumental et le discours historique sont restés plus cohérents, y compris dans l'explication des évolutions monumentales et y compris lorsqu'on a sciemment voulu privilégier une période d'âge d'or de l'établissement.

4 Nous partirons d'une définition large du «monument » : à la fois la matérialité d'un édifice ou d'un objet (amphibologie initiale) et résultat d'une action juridique (protection au titre des monuments historiques), pour revisiter celles d'« objet » et de « lieu». 
Figure 1. L'Hôpital de Tonnerre en 1293

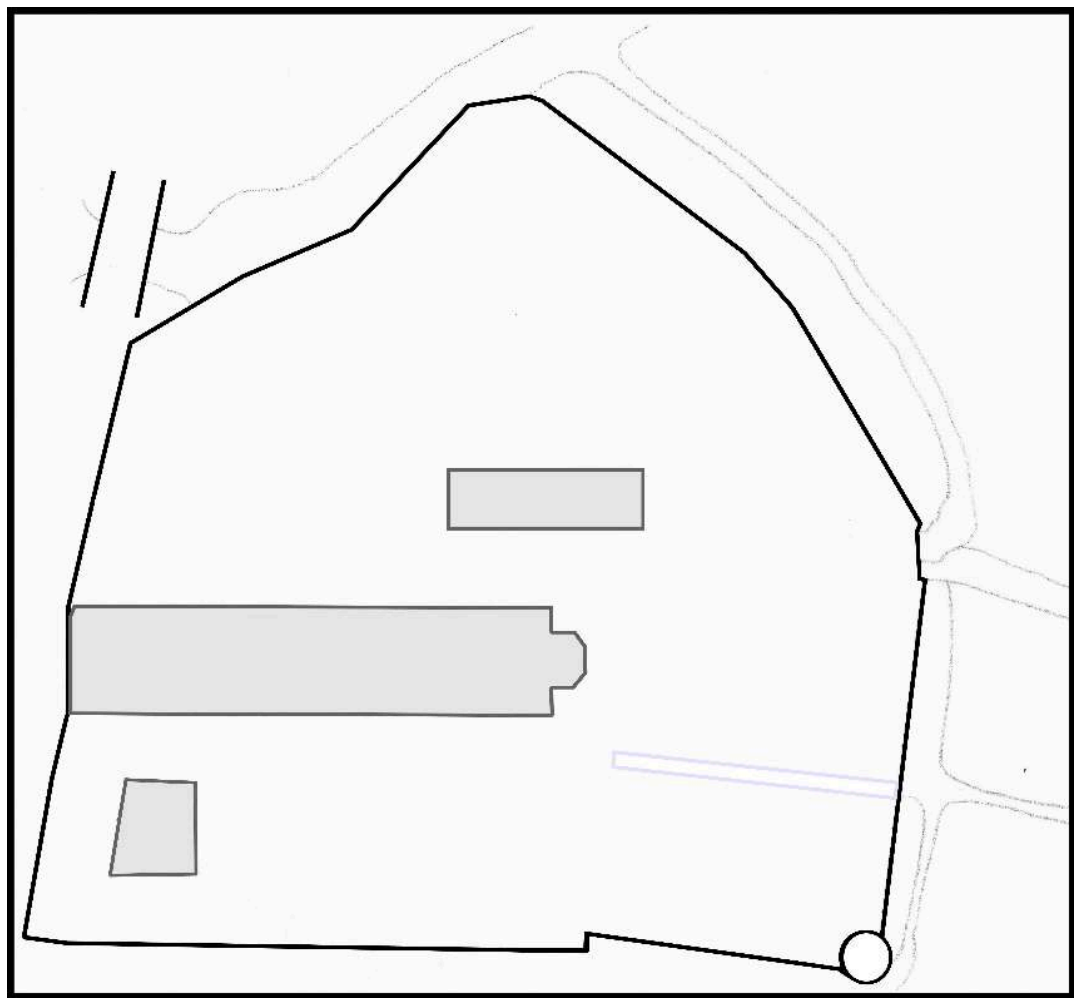

Source : Magali Villetard, Centre hospitalier de Tonnerre

Figure 2. L'Hôpital de Tonnerre au XVIII ${ }^{\mathrm{e}}$ siècle

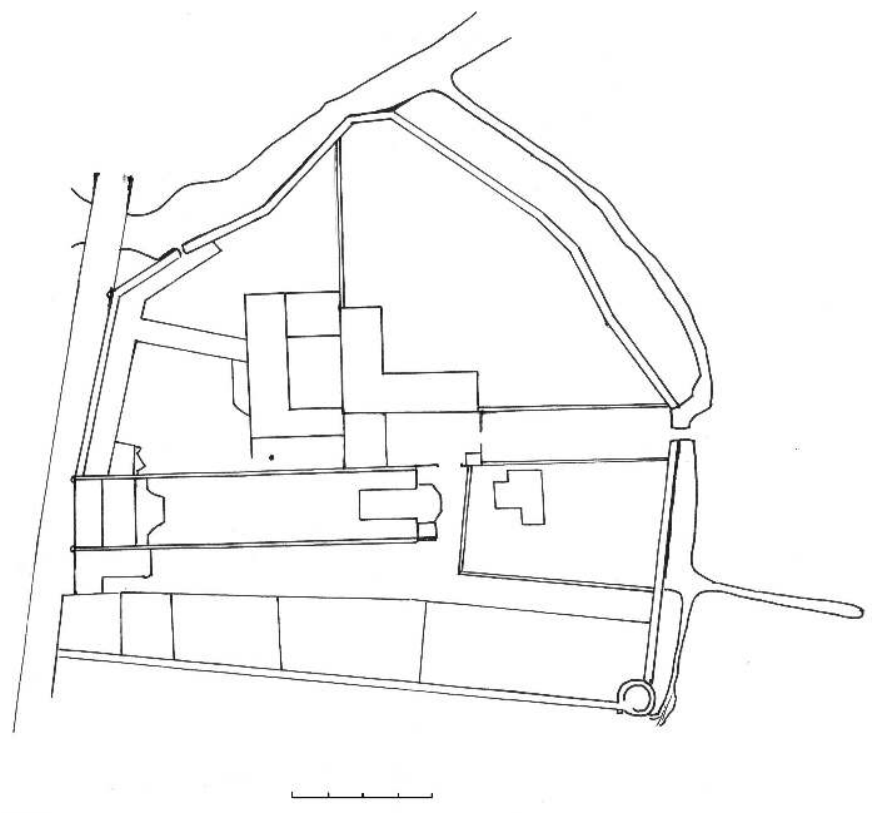

L'Hôpital de Tonnerre au XVIIIle siècle

Source : Magali Villetard, Centre hospitalier de Tonnerre 
Figure 3. L'Hôpital de Tonnerre en 1852

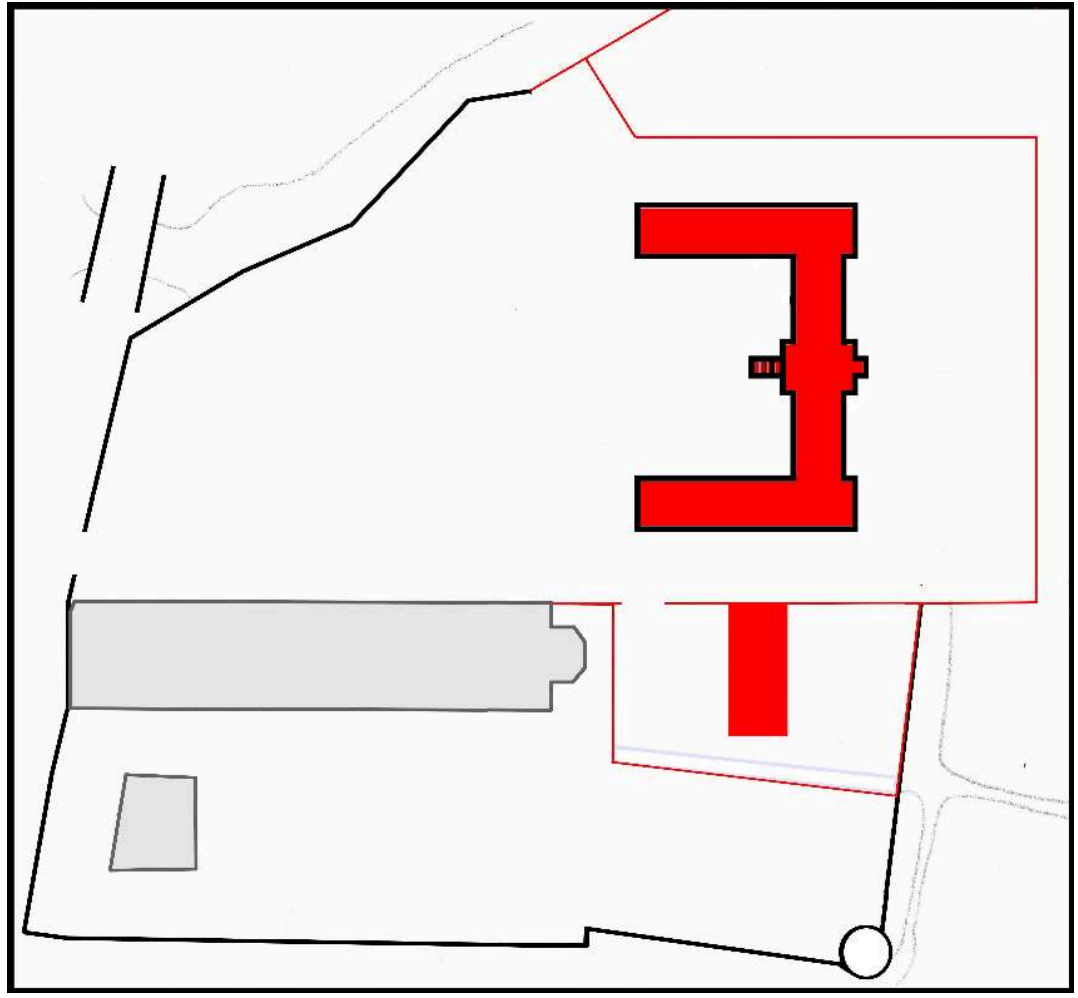

Source : Magali Villetard, Centre hospitalier de Tonnerre

Retour sur les faits : I'Hôtel-Dieu, « œuvre » de Marguerite de Bourgogne, comtesse de Tonnerre

L'Ancien Régime 
Figure 4. Vue d'ensemble façade latérale sud de l'Hôtel-Dieu médiéval

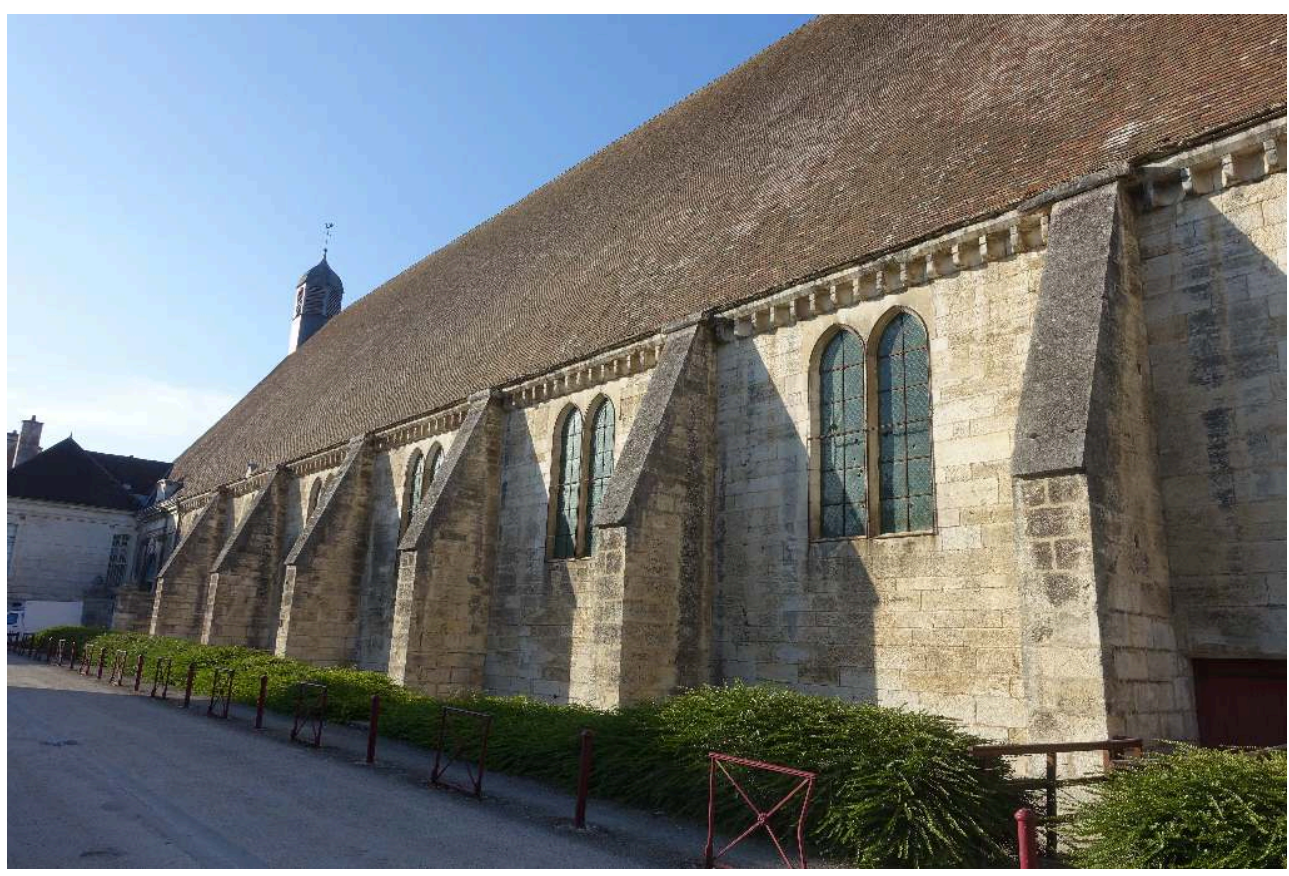

Source : Sylvie Le Clech-Charton

5 On assiste à une conception unitaire du lieu, qui s'articule autour du lien physique entre le bâtiment de soins construit à partir de 1293, qui sert aussi de lieu de culte, la domus dei, et le domaine de la comtesse et de son chapelain. En effet, le château de la comtesse est lié à l'édifice hospitalier par une galerie, et la maison du chapelain se trouve immédiatement derrière l'édifice médiéval.

6 Cette unité est mise à mal pendant la guerre de Cent Ans, puis lors des guerres de religion, périodes de grande insécurité durant lesquelles on assiste à des appropriations par des « usages » de particuliers qui considèrent en quelque sorte le bien de l'hôpital non pas comme un "commun", ce qui avait pu être le cas de figure de certains bois seigneuriaux laissés sans entretien, mais comme une portion utilisée à leur profit. L'établissement d'un plan visuel de la fin du XVI ${ }^{e}$ siècle s'impose donc pour remettre le domaine initial dans ses droits. Ce plan prouve, en particulier parce qu'il a servi jusqu'au $\mathrm{xVIII}^{\mathrm{e}}$ siècle, que la vision domaniale et symbolique unitaire prévaut alors même que la matérialité des bâtiments peut souffrir de solutions de continuité. Le domaine foncier unique est en effet la trace juridique et matérielle du dessein de la comtesse bienfaitrice de la population accueillie : accueillir, nourrir, soigner les âmes et les corps. Le « dessin » du plan représente ainsi le « dessein » de la bienfaitrice.

On distingue plusieurs périodes de construction, qui ne remettent pas en cause ce "dessein », mais l'enrichissent et le précisent, l'adaptant à des évolutions nécessaires :

- le noyau de 1293 ;

- le $\mathrm{XVI}^{\mathrm{e}}$ siècle : la disposition médiévale perdure. Une salle des malades commune classique, des circulations astucieuses, une articulation entre soin des âmes et des corps ;

- l'expansion du XVII siècle autour de la cour : dissociation des fonctions de culte et de soins ;

- les modifications de façade et extensions diverses du XVIII siècle (qui occultent la façade médiévale) ; 
- la période révolutionnaire - les $\mathrm{XIX}^{\mathrm{e}}$ et $\mathrm{Xx}^{\mathrm{e}}$ siècles (qui achèvent la déconstruction reconstruction de la perception de l'espace commun).

La Révolution attaque en partie l'unité physique, mais surtout économique et symbolique du lieu, qui change partiellement d'usage. L'ancien Hôtel-Dieu médiéval, déjà restreint à sa fonction de culte au XVII ${ }^{\mathrm{e}}$ siècle, devient un lieu de stockage du foin et se trouve vidé en grande partie de son mobilier.

7 S'agissant du mobilier et ses pérégrinations : la perception d'un lieu se fait aussi par l'existence d'un mobilier conçu pour ce lieu ou qui, même rapporté, accompagne l'usage du lieu. Notre perception actuelle, celle d'un grand vide, est influencée par l'histoire des collections de mobilier et singulièrement, par l'histoire des tombeaux des familles de bienfaiteurs.

\section{L'église rendue au culte en 1811}

8 L'importance des monuments funéraires aujourd'hui visibles est symbolique du changement de perception de la grande salle et du phénomène de sa patrimonialisation, qui fait entrer l'hôtel-Dieu dans une narration historique: initialement et symboliquement, ce qui donne une unité et un sens à ce type de lieu, conçu comme l'œuvre d'un puissant charitable, est la présence des corps des fondateurs et bienfaiteurs successifs dans les tombeaux. La tradition de se faire inhumer dans l'église est suivie par les continuateurs de Marguerite de Tonnerre, les Clermont-Tonnerre, puis les Louvois. La Révolution introduit là aussi une rupture dans la perception des lieux qui sont vidés de leurs "corps saints", ceux des propriétaires bienfaiteurs. Le monument funéraire devient progressivement la seule représentation visuelle possible d'une absence physique due aux tribulations de la fin du mois d'août 1792 (les tombeaux sont dispersés, les corps soustraits).

Durant toute la première moitié $\mathrm{du} \mathrm{XIX}^{\mathrm{e}}$ siècle, avant les grandes mesures de protection des années 1840-1913, on assiste à une démarche de reconstitution qui est un discours de représentation et de mise en scène : l'objet Hôtel-Dieu est patrimonialisé par étapes et la vision unitaire ancienne, domaniale est remplacée, pour le visiteur d'aujourd'hui, par une série d'assignations successives.

10 Le $\mathrm{xIX}^{\mathrm{e}}$ siècle marque le début d'un processus de déconstruction de l'ensemble monumental et mobilier réel, fruit du temps long, et de reconstruction symbolique sous la forme d'un objet patrimonial. Le processus a sélectionné les éléments à présenter. La protection juridique au titre des monuments historiques (cf. définition ci-dessus) donne de fait une légitimité et une crédibilité à un bâtiment et à son contenu en réalité parvenus jusqu'à nous à la suite d'adaptations à des nécessités contingentes (destructions, acquisitions, déplacements, réinstallations exogènes ou endogènes). Les bâtiments du XVII ${ }^{\mathrm{e}}$ siècle ont en effet disparu. Le château comtal médiéval puis moderne a laissé place au pavillon Dormois, du nom de l'intendant directeur historien du XIX siècle. Ce nouvel hôpital, construit entre 1848 et 1852, a été déconnecté de l'ancien Hôtel-Dieu. En fond de parcelle, la modernisation hospitalière a donné lieu à des adjonctions de modules $\mathrm{du} \mathrm{xx}^{\mathrm{e}}$ siècle, placés dans un environnement de jardins et desservis par une entrée qui est clairement détournée du centre historique. 
Si les bâtiments du XIX siècle ont conservé leurs usages hospitaliers et constituent un ensemble immobilier en dépit du choix d'implantation pavillonnaire, l'Hôtel-Dieu médiéval est donc physiquement séparé du reste par deux éléments :

- la grille qui clôt l'hôpital du xix siècle ;

- le jardin aménagé au tout début du xxe siècle.

Il a été assigné à la fonction d'entretien du souvenir de la comtesse et des bienfaiteurs, tant au rez-de-chaussée (hors musée hospitalier) qu'au premier étage (musée hospitalier fonctionnant par " period rooms »). La création du « musée » conforte donc le processus de transformation de l'Hôtel-Dieu en un objet patrimonial bicéphale :

- d'un côté le grand espace vide où les seuls éléments mobiliers sont relégués de chaque côté des murs, au niveau du chœur et dans la chapelle qui accueille la Mise au tombeau ;

- de l'autre, l'espace muséal qui reconstitue un idéal type de scènes de la vie de l'hôpital, autour des fonctions suivantes : alimentaire (la grande cuisine à l'emplacement de la salle de malades Courtanvaux du xvIII siècle et à l'emplacement de la salle d'opération), religieuse (les objets de culte et les statues de Marguerite et de l'une de ses compagnes traitées en objets d'art sous vitrine), institutionnelle (salle du conseil dans les combles), soignante (salle d'opération et chambre du malade représentant le passage à une médecine moderne au moment de la guerre de 1914, dans les combles).

11 Le discours s'incarne dans l'espace, l'histoire est mise en scène dans une vision panoramique, mais concentrée dans un lieu dont on ne comprend plus les transformations et fonctions successives.

Cette décontextualisation est observable à travers les usages $\mathrm{du} \mathrm{xx}^{\mathrm{e}}$ siècle et à travers la perception de ces usages par la population. L'ancien hôtel-Dieu est un lieu commun de rassemblement pour des manifestations commerciales et culturelles (salons, représentations, colloques). L'hôpital moderne suit son chemin d'établissement de soins.

\section{L'état des recherches sur l'hôpital de Tonnerre}

\section{La fonction unificatrice de Camille Dormois, directeur, archiviste et historien}


Figure 5. Pièce au $1^{\text {er }}$ étage, susceptible d'avoir abrité les archives de l'ancien hôtel-Dieu médiéval

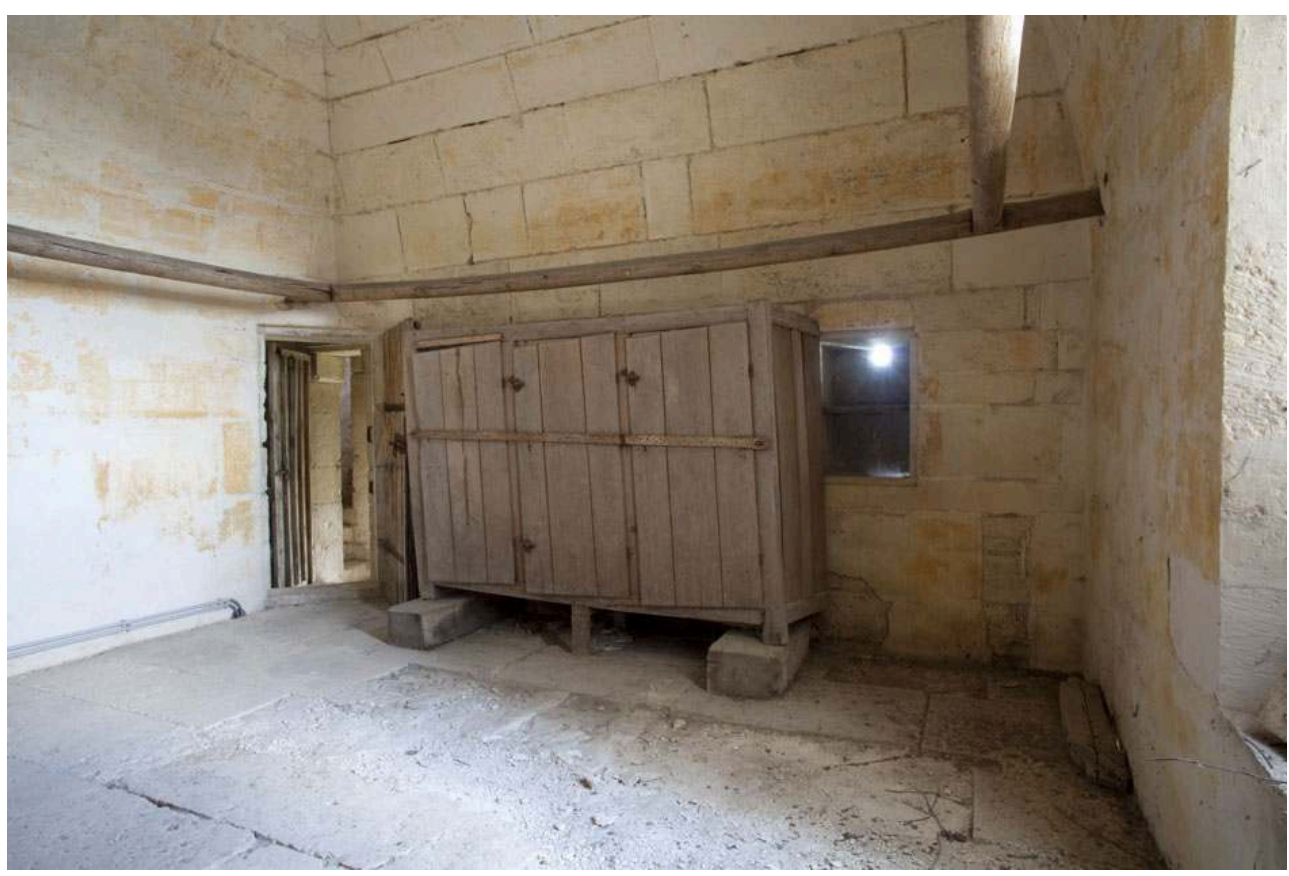

Source : Sylvie Le Clech-Charton

13 Camille Dormois a écrit, à partir des archives, mais aussi en faisant référence à de la littérature de seconde main ancienne et à des souvenirs oraux, une histoire de l'hôpital qui conforte une continuité institutionnelle et reste donc fidèle à l'intention domaniale de départ : fonder un domaine dont les pauvres seraient les principaux bénéficiaires et pour toujours. Ce discours domanial donne ses moyens économiques et juridiques aux politiques de l'assistance sur la longue durée et malgré les changements radicaux de régimes politiques. Il permet de faire perdurer juridiquement la «fondation» médiévale, initiée dans un contexte juridique et spirituel totalement différent. Il lui assure donc ses effets dans le temps présent. Il permet enfin de surmonter les dissociations monumentales et patrimoniales qui aboutissent de fait à traiter l'HôtelDieu comme un objet. Son rôle est fondamental, puisqu'il fait le trait d'union entre la période révolutionnaire et la nôtre.

\section{Les apports des $\mathrm{XX}^{\mathrm{e}}$ et $\mathrm{XX} \mathrm{I}^{\mathrm{e}}$ siècles}


Figure 6. Journée d'étude Passé/Présent

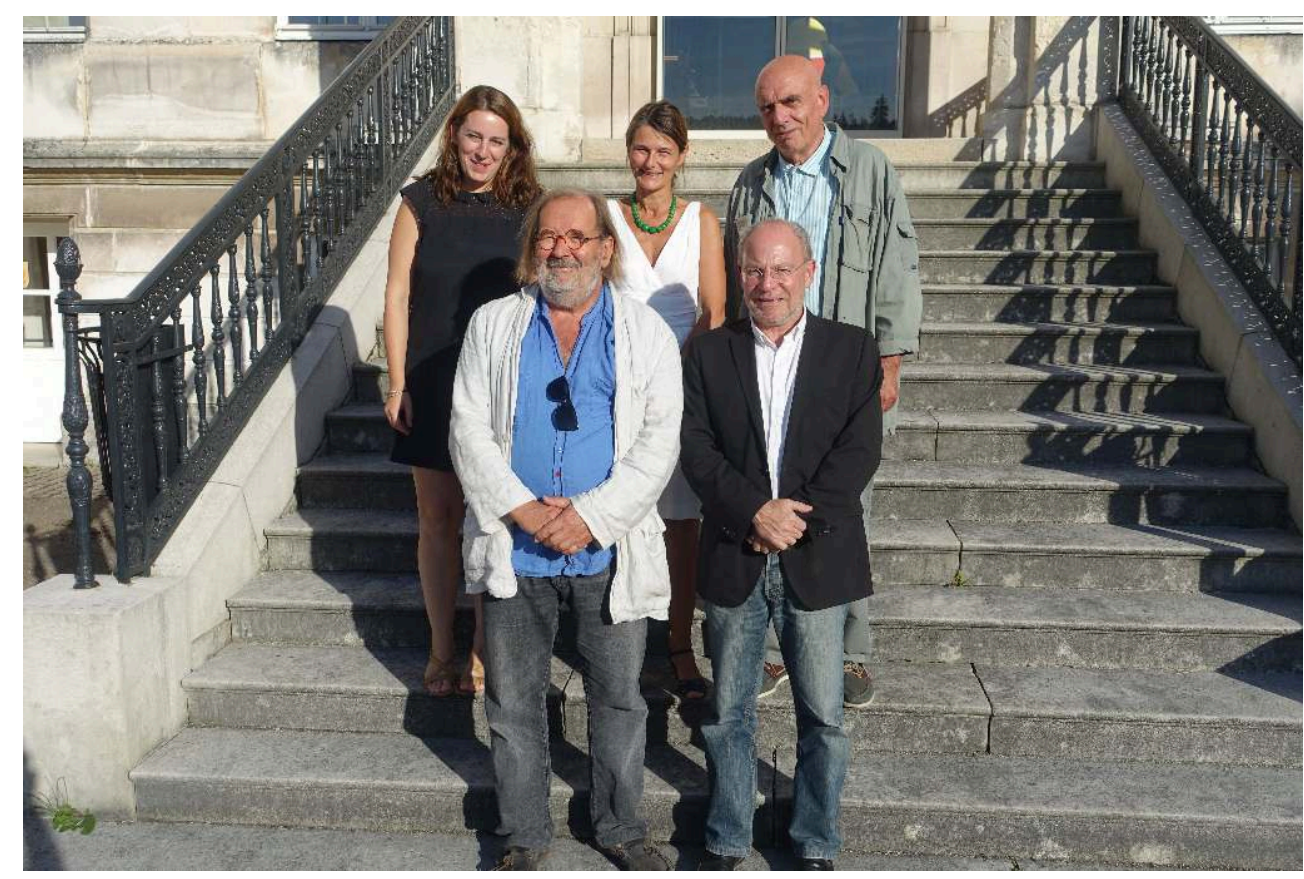

Hôpital de Tonnerre, 10 septembre 2016

Source : Sylvie Le Clech-Charton

Dans la grande tradition des études locales hospitalières, les années 1950-1970 ont vu se développer des recherches qui ont classiquement évolué dans deux directions: les monographies d'érudition locale, les premiers travaux universitaires qui, partant des archives, ont surtout traité l'œuvre charitable sous ses aspects institutionnels, juridiques et économiques. La focalisation sur le personnage de Marguerite de Tonnerre est le fruit d'une tendance constante de l'historiographie depuis le xIX $x^{e}$ siècle, et de la constitution d'un savoir historique professionnel en France.

Elle rejoint là l'importance mémorielle du personnage de la comtesse, vue comme un élément sociologique essentiel qui fait la jonction entre une histoire régionaliste (la Bourgogne) et nationale (celle de la France). Le personnage de la comtesse humanise et met en valeur une histoire collective et territoriale.

Quand les premiers travaux de restauration commencent et s'intensifient (campagnes de restauration de Bernard Colette, 1990 et suivantes), le savoir historique est pris tel quel et les seuls apports scientifiques sont constitués par l'archéologie et les fouilles qui ne sont pas encore préventives (régime antérieur à la loi sur l'archéologie de 2001). Le savoir historique est davantage traité comme une toile de fond et les dossiers de travaux s'attachent plus à suivre les doctrines successives diffusées dans les milieux professionnels de la restauration du patrimoine.

Bien que les actions des services patrimoniaux s'attachent à redonner au monument son unité d'œuvre, en assumant des risques intellectuels classiques (le choix de la période de référence, la mise en valeur de la charpente...) et donc à stopper le processus de transformation en objet, elles se heurtent toujours au vide créé et sans doute subi de l'espace de l'ancien Hôtel-Dieu. Celui-ci est en effet dépourvu de certains objets dont on ne connait le stock initial et mouvant que par la consultation des inventaires successifs. Faute de lien entre l'interprétation des données sur les objets et leur spatialisation 
aujourd'hui, le réaménagement contemporain n'exploite en réalité pas les données des inventaires qui replacent les objets dans les pièces où ils avaient leur utilité. L'aspect visuel de l'ancien Hôtel-Dieu est typique de l'insuffisance de lien entre le contenu et le contenant. Ces insuffisances sont des symptômes de savoirs professionnels dissociés en fonction des cultures techniques et figés par une législation patrimoniale conservatrice. Il a fallu la loi Liberté de Création Architecture et Patrimoine, promulguée le 7 juillet 2016, pour arriver à concevoir un lien historique entre une série d'objets conçue pour un monument et le monument lui-même. La notion d'ensemble historiquement attachée au monument permet de définir, a contrario, les cas où les ensembles ou sous-ensembles sont rapportés dans le monument ou soustraits par les vicissitudes de l'histoire. La conséquence opérationnelle possible d'application de cette loi est un réaménagement des lieux en fonction d'un choix à deux entrées complémentaires. La première entrée est celle de la critique historique des objets et de leur emplacement initial. Elle aboutit à présenter un parcours unifié d'objets tant civils (ceux conçus pour la cuisine par exemple ou ceux des salles de chirurgie du $\mathrm{xVIII}^{\mathrm{e}}$ au xx siècle) que religieux présents durant la vie du monument et incarnant ses fonctions, et ce sur les deux étages. Aujourd'hui, le visiteur ne peut comprendre que le rez-dechaussée de l'édifice vide qu'il traverse contient pourtant des objets au titre du code du patrimoine, qui forment un ensemble avec ceux présentés hors contexte au premier étage et dans les combles. La nature globale de la compréhension en est donc affectée. La seconde entrée est performative. L'entrée artistique et créative permet de s'abstraire des réaménagements matériels d'une présentation ancienne qui, pour quantité de raisons, ne pourrait être totalement changée. La création numérique visuelle et sonore, y compris par des procédés d'immersion du visiteur dans une autre dimension met en œuvre des émotions mais aussi des perceptions cognitives qui peuvent favoriser une appréhension de l'unité d'œuvre que constituent l'édifice et son contenu. Sur un plan plus matériel, la «fabrique " possible de pavillons temporaires peut constituer autant de boîtes dans la boîte de l'édifice lui-même. Leur construction peut témoigner d'une compréhension architecturale des lieux tels que transmis aujourd'hui. Sont ainsi exploités les pleins et les vides, le convexe et le concave, la lumière et son circuit tout au long du jour. Le travail de l'architecte permet un autre travail de l'esprit. Le « dessein » des maîtres d'œuvre et des bienfaiteurs historiques du lieu peut y être présenté de manière pédagogique, créative, à la manière de chambres photographiques à hauteur d'hommes. Ainsi, une unité d'œuvre malmenée par sa propre histoire, que constitue la «fondation" juridique et pieuse de la comtesse se trouve-t-elle réincarnée et transmise, mais non figée. La création de telles architectures provisoires permet, par la notion de " projet », de faire participer les habitants qui, sur le territoire, ont tous un "point de vue " sur l'hôtel-Dieu, patrimoine matériel, juridique, social et symbolique. L'architecture, qui a créé des bâtiments patrimoniaux dans le passé, peut ainsi créer des "lieux" au $\mathrm{xxI}^{\mathrm{e}}$ siècle, comme le démontre la Biennale d'architecture de Venise en cette année 2018. 


\section{BIBLIOGRAPHIE}

Le Clech-Charton Sylvie, L'Hôtel-Dieu de Tonnerre. Métamorphose d'un patrimoine hospitalier, $\mathrm{XIII}^{e}-\mathrm{XX}{ }^{e}$ siècle, Langres, Éditions Dominique Guéniot, 2012. L'ouvrage comporte une importante bibliographie.

INDEX

Mots-clés : Monument, œuvre, patrimoine, musée, décontextualisation

\section{AUTEUR}

\section{SYLVIE LE CLECH-CHARTON}

Sylvie Le Clech-Charton est archiviste paléographe, historienne, conservatrice générale du patrimoine, Inspectrice générale des Patrimoines et directrice de la DRAC Centre-Val de Loire (2013-2018.) 